\title{
Two Algorithms for Model Quality Estimation in State-Space Systems with Time-Varying Parameter Uncertainty
}

\author{
Soheil Salehpour and Andreas Johansson
}

\begin{abstract}
We present two methods to estimate bounds of parameter uncertainty in state-space systems. In the first method, we minimize the $l_{\infty}$-norm of the perturbation and its derivative. In the second method, an estimate of the perturbation is produced based on a quantized approximation of the uncertainty and the sparse structure of its derivative. Less sensitivity to increased noise and changed model parameters is achieved by the second method. We use an overhead crane as an illustrative example.

Index Terms-Model quality estimation, time-varying parameter uncertainty, MILP, sparsity, perturbation, uncertainty, optimization.
\end{abstract}

\section{INTRODUCTION}

$\mathbf{T}$ HE uncertainties associated with the nominal process model is a concern in most approaches to feedback control. The question is how to achieve a tight bound or shape of the uncertainty by using a set of measurement data. This active research area is known as model quality estimation. In the existing approaches to model quality estimation, the true system, see e.g. [1], [2], [3], [4] and [5], is a linear time-invariant system (where uncertainty is considered in both $H_{\infty}$ and $L_{1}$ ). A time varying linear system is a more realistic assumption, since nonlinear behavior can then also be accounted for. However, model quality estimation of time-varying perturbations appears to be difficult. Here, we consider a linear system with time-varying parameters as the model uncertainty. The prime drawback of assuming a parametric, time-varying uncertainty description is its possible shortcoming for describing unmodeled dynamics. However, if the process physics is reasonably well-known, then the unmodeled dynamics can be limited to high frequencies, which then can often be described by a parametric model.

We will assume a state-space system with affine dependence on the uncertain time-varying parameters. This structure is frequently used in robust control and estimation [9] and [10], and can be expressed by using the Kronecker product as [6]

$$
\begin{aligned}
x(k+1)= & A x(k)+B u(k)+P(\pi(k) \otimes x(k))+ \\
& D \pi(k)+Q(\pi(k) \otimes u(k)) \\
y(k)= & C x(k)+\eta(k)
\end{aligned}
$$

where $\otimes$ denote the Kronecker product and $A \in R^{n \times n}, B \in$ $R^{n \times p}, C \in R^{l \times n}, D \in R^{n \times q}, P \in R^{n \times n q}, Q \in R^{n \times q p}$ are constant matrices. The vector $x(k) \in R^{n}$ represents the state, $y(k) \in R^{l}$ is the measured output and $u(k) \in R^{p}$ is the input while $\eta(k) \in R^{l}$ is some additive disturbance. The

Andreas Johansson and Soheil Salehpour are with the Control Engineering Group, Luleå University of Technology, SE-971 87 Luleå, Sweden.

soheil@ltu.se, andreas.johansson@ltu.se vector $\pi(k) \in R^{q}$ represents the parameter uncertainties, i.e. the deviation from the nominal parameter values. Note that they are allowed to enter both linearly and and bilinearly with the state $x$ as well as with the input $u$.

Here, we will present two methods for estimating upper bounds for the uncertainties $\left(|\pi(k)| \leq \rho \in R^{q}\right)$. The first method minimizes the $l_{\infty}$-norm of the uncertainty and its derivative, and the second method is based on optimization of the sparsness of the derivative of the quantized perturbation.

An approximation of (1) is given in Section II. In Section III, we define a bound for the disturbance. An $l_{\infty}$ optimization method is presented in Section IV. A MILP (Mixed Integer Linear Programming) algorithm to minimize the sparsity of a matrix is introduced in Section V, and in Section VI, we use the MILP-optimization method to estimate the perturbation. An overhead crane process is represented in Section VII, and the methods are applied to this process in Section VIII. Section IX gives some concluding remarks and directions for future work.

\section{The Biaffine InPut/Output System}

In this section, we give an approximation of (1) whose output is affine in the input $u$ and perturbation $\pi$. It is derived in [11] as a 1'st order Taylor approximation of $y$ with respect to $\pi$ and may be expressed as

$$
\begin{aligned}
\zeta(k+1)= & A \zeta(k)+B u(k) \\
\xi(k+1)= & A \xi(k)+P(\pi(k) \otimes \zeta(k))+ \\
& D \pi(k)+Q(\pi(k) \otimes u(k)) \\
\hat{y}(k)= & C(\zeta(k)+\xi(k))
\end{aligned}
$$

with initial state $\zeta(0)=x(0)$ and $\xi(0)=0$. By defining the disturbance $\nu=r+\eta$, where $r$ denotes the linearization error, the output of (1) may be expressed as

$$
y(k)=\hat{y}(k)+\nu(k)
$$

Furthermore, by collecting the signals into vectors as

$$
\begin{aligned}
& \Pi=\left[\pi(0)^{T} \pi(1)^{T} \cdots \pi(N-1)^{T}\right]^{T} \\
& Y=\left[y(0)^{T} y(1)^{T} \cdots y(N)^{T}\right]^{T} \\
& V=\left[\nu(0)^{T} \nu(1)^{T} \cdots \nu(N)^{T}\right]^{T}
\end{aligned}
$$

(2) may also be formulated [11] as

$$
Y=\Upsilon+\Xi \Pi+V
$$

where $\Xi=\Omega+\Psi+\Phi$, and $\Omega, \Phi, \Psi$ and $\Upsilon$ are defined in (3). 


$$
\begin{aligned}
& \Omega=\left[\begin{array}{cccc}
0 & 0 & \cdots & 0 \\
C P\left(I_{q} \otimes \zeta(0)\right) & 0 & \cdots & 0 \\
C A P\left(I_{q} \otimes \zeta(0)\right) & C P\left(I_{q} \otimes \zeta(1)\right) & \cdots & 0 \\
\vdots & \vdots & \ddots & \vdots \\
C A^{N-2} P\left(I_{q} \otimes \zeta(0)\right) & C A^{N-3} P\left(I_{q} \otimes \zeta(1)\right) & \ddots & 0 \\
C A^{N-1} P\left(I_{q} \otimes \zeta(0)\right) & C A^{N-2} P\left(I_{q} \otimes \zeta(1)\right) & \cdots & C P\left(I_{q} \otimes \zeta(N-1)\right)
\end{array}\right] \\
& \Phi=\left[\begin{array}{cccc}
0 & 0 & \cdots & 0 \\
C Q\left(I_{q} \otimes u(0)\right) & 0 & \cdots & 0 \\
C A Q\left(I_{q} \otimes u(0)\right) & C Q\left(I_{q} \otimes u(1)\right) & \cdots & 0 \\
\vdots & \vdots & \ddots & \vdots \\
C A^{N-2} Q\left(I_{q} \otimes u(0)\right) & C A^{N-3} Q\left(I_{q} \otimes u(1)\right) & \ddots & 0 \\
C A^{N-1} Q\left(I_{q} \otimes u(0)\right) & C A^{N-2} Q\left(I_{q} \otimes u(1)\right) & \cdots & C Q\left(I_{q} \otimes u(N-1)\right)
\end{array}\right] \\
& \Psi=\left[\begin{array}{cccc}
0 & 0 & \cdots & 0 \\
C D & 0 & \cdots & 0 \\
C A D & C D & \cdots & 0 \\
\vdots & \vdots & \ddots & \vdots \\
C A^{N-2} D & C A^{N-3} D & \ddots & 0 \\
C A^{N-1} D & C A^{N-2} D & \cdots & C D
\end{array}\right], \Upsilon=\left[\begin{array}{c}
C \zeta(0) \\
C \zeta(1) \\
\vdots \\
C \zeta(N)
\end{array}\right]
\end{aligned}
$$

\section{Disturbance Bound}

To bound the disturbance $\nu(k)$ we will use the window norm, which for continuous time signals is defined as [13]

$$
\|\nu\|_{\omega}=\sup _{t \geq 0} \int_{0}^{t} \omega(t-\tau)|\nu(\tau)| d \tau
$$

where $\omega(t)$ is nonnegative and bounded by an exponentially decreasing function. In [11] a discrete time window norm is defined as follows

Definition 1: A window sequence is a sequence $\omega \in Z^{+} \rightarrow$ $R^{+}$, which is not identically zero and satisfies $\omega(k) \leq c e^{-a k}$ for all $k \geq 0$ and some positive $c$ and $a$.

Given a window sequence $\omega(k)$, the window norm for a discrete time signal $v(k)$ may then be defined as [11]

$$
\|\nu\|_{\omega}=\sup _{k \geq 0} \sum_{i=0}^{k} \omega(k-i)|\nu(i)|
$$

It is remarked that (5) satisfies all properties of a norm, but the proof is omitted. It is straightforward to see that the window norm is equal to the $l_{\infty}$-norm by choosing $\omega$ as the unit pulse function. The drawback of $l_{\infty}$-norm is that it only considers the peak value of the signal without any averaging and may therefore be conservative. This problem is handled in the window norm by choosing a window function that averages over a suitable time interval, i.e. a pulse function with non-unit duration or a decaying exponential function. The window norm actually approaches the $l_{1}$-norm by letting $\omega$ approach a unit step function. An assumption on the disturbance $\nu=\left[\nu_{1}, \ldots, \nu_{l}\right]$ may now be expressed as

$$
\left\|\nu_{j}\right\|_{\omega} \leq \epsilon_{j}, j=1, \ldots, l
$$

We assume that each $\omega(k)$ is monotone decreasing for $k>0$. Then, as showed in [11] for scalar $\nu$, the condition (6) can be expressed as

$$
W|V| \leq 1_{N+1} \otimes \epsilon
$$

where

$$
W=\left[\begin{array}{cccc}
\omega(0) & 0 & \cdots & 0 \\
\omega(1) & \omega(0) & \cdots & 0 \\
\vdots & \vdots & \ddots & \vdots \\
\omega(N) & \omega(N-1) & \cdots & \omega(0)
\end{array}\right]
$$

where $\omega=\operatorname{diag}\left(\omega_{1}, \omega_{2}, \ldots, \omega_{l}\right)$ and $\epsilon=\left[\epsilon_{1}, \epsilon_{2}, \ldots, \epsilon_{l}\right]^{T}$

\section{THE $l_{\infty}$-OPTIMIZATION METHOD}

We assume that the purturbation is small and slowly varying and that the disturbance $\nu$ is also small. Here, we try to find the tightest bound for the perturbation. The size of the perturbation $\pi$ is measured with $\sup _{k}\|F \pi(k)\|_{\infty}$, where $\|\cdot\|_{\infty}$ is the $l_{\infty}$ vector norm and $F$ is a diagonal weight matrix. The time-derivative of $\pi$ is approximated by $(\Delta \pi)(k)=\pi(k)-\pi(k-1)$ and its size is measured with $\sup _{k}\|G \Delta \pi(k)\|_{\infty}$, where $G$ is a diagonal weight matrix.

Scaling of the vectorized perturbation $\Pi$ and its derivative $\Delta \Pi=\left[\Delta \pi(0)^{T} \Delta \pi(1)^{T} \cdots \Delta \pi(N-1)^{T}\right]^{T}$ is accomplished by

$$
\begin{aligned}
(I \otimes F) \Pi & =\left[(F \pi(0))^{T}(F \pi(1))^{T} \ldots(F \pi(N-1))^{T}\right]^{T} \\
(I \otimes G) \Delta \Pi & =\left[(G \Delta \pi(0))^{T}(G \Delta \pi(1))^{T} . .(G \Delta \pi(N-1))^{T}\right]^{T}
\end{aligned}
$$

The $l_{\infty}$-optimization problem is formulated as follows:

$$
\begin{array}{rc}
\underset{\Pi, \epsilon}{\operatorname{minimize}} & \|(I \otimes F) \Pi\|_{\infty}+\|(I \otimes G) \Delta \Pi\|_{\infty}+h \epsilon \\
\text { subject to: } & W\left|Y_{m}-\Upsilon-\Xi \Pi\right| \leq 1_{N+1} \otimes \epsilon
\end{array}
$$


where $Y_{m}=\left[y_{m}(0)^{T} y_{m}(1)^{T} \cdots y_{m}(N)^{T}\right]^{T}$, and $y_{m}(k)$ is the measured outputs of (1) for $k=0, \cdots, N$ and $h$ is a weight for the disturbance.

\section{SPARSE MATRiX}

In numerical analysis, a sparse matrix is a matrix populated primarily with zeros. The concept of sparsity is useful in complex systems and many application areas such as network theory. Huge sparse matrices often appear in science or engineering when solving partial differential equations.

One common approach to seeking a sparse description is based on $l_{1}$-norm regularization [7] which produces an approximation with a sparse structure.

In this article, we present an exact solution of sparsity by using MILP (Mixed Integer Linear Programming) to minimize the number of non-zero elements in a matrix or vector.

We consider the logical variable $\delta_{i j} \in\{0,1\}$ and the matrix $S=\left(s_{i j}\right)_{i, j=1 \ldots N}$ to be related as

$$
\begin{aligned}
& \delta_{i j}=1 \leftrightarrow s_{i j} \neq 0 \\
& \delta_{i j}=0 \leftrightarrow s_{i j} \equiv 0
\end{aligned}
$$

and we aim to minimize $\sum_{i, j=1}^{N} \delta_{i j}$ for $i, j=1, \ldots, N$. First, we assume $\delta_{i j}=\delta_{i j}^{1}+\delta_{i j}^{2}$ and (9) is re-written as

$$
\begin{aligned}
& \left(\delta_{i j}^{1}, \delta_{i j}^{2}\right)=(1,0) \leftrightarrow s_{i j}>0 \\
& \left(\delta_{i j}^{1}, \delta_{i j}^{2}\right)=(0,0) \leftrightarrow s_{i j}=0 \\
& \left(\delta_{i j}^{1}, \delta_{i j}^{2}\right)=(0,1) \leftrightarrow s_{i j}<0
\end{aligned}
$$

We establish a more practical link between logical and real variables by

$$
\begin{aligned}
m \delta_{i j}^{1}-M \delta_{i j}^{2} & \leq s_{i j} \leq M \delta_{i j}^{1}-m \delta_{i j}^{2} \\
\delta_{i j}^{1}+\delta_{i j}^{2} & \leq 1
\end{aligned}
$$

where $m=\min _{i, j}\left(\left|s_{i j}\right|\right)$ and $M=\max _{i, j}\left(\left|s_{i j}\right|\right)$. Then, a MILP for maximizing sparsity of $S$ is formulated as

$$
\begin{array}{cc}
\operatorname{minimize} & \sum_{i, j=1}^{N}\left(\delta_{i j}^{1}+\delta_{i j}^{2}\right) \\
\text { subject to: } & (10)
\end{array}
$$

Here, we also present an approximaion method which is more practical in a numerical sense. This method instead minimizes the number of elements of a matrix which are larger than a certain threshold. If we consider $\mu$ as threshold, the logical variables are changed into

$$
\begin{aligned}
& \delta_{i j}=1 \leftrightarrow\left|s_{i j}\right|>\mu \\
& \delta_{i j}=0 \leftrightarrow\left|s_{i j}\right| \leq \mu
\end{aligned}
$$

Then, the inequalities in (10) are substituted in (11) by

$$
\begin{aligned}
2 \mu \delta_{i j}^{1}-M \delta_{i j}^{2}-\mu & \leq s_{i j} \leq M \delta_{i j}^{1}-2 \mu \delta_{i j}^{2}+\mu \\
\delta_{i j}^{1}+\delta_{i j}^{2} & \leq 1
\end{aligned}
$$

where $M=\max _{i, j}\left(\left|s_{i j}\right|\right)+\mu$.

\section{Method BASEd on MILP AND The DeRIVATIVE OF UNCERTAINTY}

Quantization as a means of approximating signals is widely used in digital control and other areas. The differentiation of a quantized signal produces a lot of zero samples and thus, it has a sparse structure.

Our basic assumption is that the perturbations are due to deterministic physical phenomenae and thus have a nonrandom nature. In particular, we assume that the perturbations can be approximated by quantization. Then, the following sparsity property of its derivative is used for estimating the uncertainties and bounds for them. With the definitions

$$
\begin{aligned}
\sigma_{i} & =\left[\delta_{1 i}^{1}, \delta_{1 i}^{2}, \cdots, \delta_{q i}^{1}, \delta_{q i}^{2}\right] \text { for } i=0, \cdots, N-1, \\
\Sigma_{N} & =\left[\sigma_{0}, \sigma_{1}, \cdots, \sigma_{N-1}\right]^{T}, U=I_{N q} \otimes[M,-2 \mu], \\
L & =I_{N q} \otimes[2 \mu,-M] \text { and } \Lambda=I_{N q} \otimes[1,1]
\end{aligned}
$$

the sparsity constraint (12) applied to the derivative $\Delta \Pi$ may be expressed as

$$
\begin{aligned}
L \Sigma_{N}-1_{N q} \mu & \leq \Delta \Pi \leq U \Sigma_{N}+1_{N q} \mu \\
\Lambda \Sigma_{N} & \leq 1_{N q}
\end{aligned}
$$

We use the constraint (13), and MILP-optimization is formulated as

$$
\begin{array}{cc}
\underset{\Sigma_{N}, \Pi}{\operatorname{minimize}} & 1_{2 N q} \Sigma_{N}+\|(I \otimes F) \Pi\|_{\infty} \\
\text { subject to: } & (13) \\
& W\left|Y_{m}-\Upsilon-\Xi \Pi\right| \leq 1_{N+1} \otimes \epsilon
\end{array}
$$

where we use the $\epsilon$ which is computed in (8) to increase the accuracy in this algorithm. Alternatively, $\epsilon$ may be computed using the sample $l_{1}$-norm method in [12].

\section{Application to a Pendulum Process}

We will apply the two methods to an overhead crane process with the position of the load $x_{p}$ as measurement $y_{c}$. The process is modeled as a pendulum where the horizontal velocity of the suspension point is proportional to the control signal $u$. With $x_{s}$ as the position of the suspension point the linearized process is formulated as

$$
\begin{aligned}
{\left[\begin{array}{l}
\dot{x}_{p} \\
\ddot{x}_{p} \\
\dot{x}_{s}
\end{array}\right] } & =\left[\begin{array}{ccc}
0 & 1 & 0 \\
-g / l & -c / m & g / l \\
0 & 0 & 0
\end{array}\right]\left[\begin{array}{l}
x_{p} \\
\dot{x}_{p} \\
x_{s}
\end{array}\right]+\left[\begin{array}{l}
0 \\
0 \\
b
\end{array}\right] u \\
y_{c} & =\left[\begin{array}{lll}
1 & 0 & 0
\end{array}\right]\left[\begin{array}{lll}
x_{p} & \dot{x}_{p} & x_{s}
\end{array}\right]^{T}
\end{aligned}
$$

where $g$ is the acceleration of gravity, $l$ is the length of the pendulum, $c$ is a friction coefficient, and $b$ is the proportionality factor converting the control signal into the speed of the suspension point. The friction coefficient is not exactly known and will vary with the speed of the load. The length of the pendulum $l$ will also vary and we assume that the proportionality factor $b$ is poorly known and possibly timevarying. We introduce the notations $\lambda=g / l$ and $\kappa=c / m$ which are thus functions of time. 
A simple Euler approximation gives the following discrete-time system

$$
\begin{aligned}
x(k+1)= & {\left[\begin{array}{ccc}
1 & h & 0 \\
-h \lambda(k) & 1-h \kappa(k) & h \lambda(k) \\
0 & 0 & 1
\end{array}\right] x(k)+} \\
& {\left[\begin{array}{c}
0 \\
0 \\
h b(k)
\end{array}\right] u_{c}(k) } \\
y(k)= & {\left[\begin{array}{lll}
1 & 0 & 0
\end{array}\right] x(k) }
\end{aligned}
$$

where $u_{c}(k)=u(k h)$ and $y(k)=y_{c}(k h)$ while $\eta(k)$ is supposed to capture both the continuous-time noise term $\eta_{c}(k h)$ and the error introduced by the approximation.

The sampling interval in our simulation is $h=0.05$ seconds. Nominal values of $\lambda, \kappa$ and $b$ and time-varying deviations from these are introduced as

$b(k)=b_{0}+\pi_{1}(k), \lambda(k)=\lambda_{0}+\pi_{2}(k), \kappa(k)=\kappa_{0}+\pi_{3}(k)$

Thus, with the definitions

$$
\begin{aligned}
A & =\left[\begin{array}{ccc}
1 & h & 0 \\
-h \lambda_{0} & 1-h \kappa_{0} & h \lambda_{0} \\
0 & 0 & 1
\end{array}\right], B=\left[\begin{array}{c}
0 \\
0 \\
h b_{0}
\end{array}\right] \\
P & =\left[\begin{array}{ccccccccc}
0 & 0 & 0 & 0 & 0 & 0 & 0 & 0 & 0 \\
0 & -h & 0 & -h & 0 & h & 0 & 0 & 0 \\
0 & 0 & 0 & 0 & 0 & 0 & 0 & 0 & 0
\end{array}\right] \\
D & =\left[\begin{array}{lll}
0 & 0 & 0 \\
0 & 0 & 0 \\
0 & 0 & 0
\end{array}\right], Q=\left[\begin{array}{ccc}
0 & 0 & 0 \\
0 & 0 & 0 \\
0 & 0 & h
\end{array}\right]
\end{aligned}
$$

the process may be expressed as (1).

Since the process is oscillating, the disturbance $\eta$ is dominated by the oscillation frequency. The window function $\omega$ in the norm $\|\cdot\|_{\omega}$ is thus chosen as a pulse function whose duration is one half oscillation period to provide averaging of the disturbance. This means

$$
\begin{gathered}
\omega(k)=\left\{\begin{array}{cl}
1 / 35 & 0 \leq k \leq 35 \\
0 & \text { otherwise }
\end{array}\right. \\
\text { VIII. SIMULATION RESULTS }
\end{gathered}
$$

To give some idea of the performance of the algorithms, we apply them to the pendulum process as follows. The output of (1) and (2) with an added white noise disturbance $\eta$ and the parameter perturbation is compared in Fig. 1 (lower). The perturbations are also depicted in Fig. 1 (upper) where $\lambda_{0}, \kappa_{0}$ and $b_{0}$ are chosen as 3.3, 0.25 and 2, respectively. The $l_{\infty}$ - and MILP-optimization methods are implemented in the YALMIP software [8] and applied to the pendulum process. The weighting factors are chosen as $F=G=$ $\operatorname{diag}\left(1 / b_{0}, 1 / \lambda_{0}, 1 / \kappa_{0}\right)$ and $h=1 /\left\|y_{m}-C \zeta\right\|_{\omega}$ in (8) for the $l_{\infty}$-method, and $F=\operatorname{diag}\left(1 / b_{0}, 1 / \lambda_{0}, 1 / \kappa_{0}\right)$ in (14) for the MILP-method.

The result of a simulation for the two methods is depicted in Fig. $2\left(\lambda_{0}=3.3, b_{0}=2, \kappa_{0}=0.25\right)$, which shows that although $l_{\infty}$-method estimates a tighter bound for the perturbution $\pi_{2}$, the MILP-method exhibits a better tracking of the true perturbation. To illustrate the sensitivity of the methods to the magnitude of the perturbation, four test cases have been considered. The shape of the parameter perturbations of the four cases is depicted in Fig. 1 (upper), but the amplitude is given by Table I. It is seen that the $l_{\infty}$-method in many cases estimates tighter bounds for the perturbation but on the other hand frequently under-estimates the bound.

The sensitivity of the algorithms to the changing of the parameters $\lambda_{0}, b_{0}$ and $\kappa_{0}$ are showed in Table II. The sensitivity of the methods to different noise levels is illustrated in Table III for different $\epsilon$ which is the window norm of the white noise. As noticed in Tables II and III, the estimated bounds for the $l_{\infty}$-method are more sensitive to the changing of $\lambda_{0}$, $\kappa_{0}, b_{0}$ and the noise level $\epsilon$. The sensitivity of the first algorithm to different parameters may be accounted to the fact that it does not consider the properties of the perturbations, e.g. the assumption that the derivative is sparse. Furthermore, the estimated perturbations are very often oscillating (Fig. 2 to Fig. 6), which is caused by the $l_{\infty}$-norm inherent property to limit the upper-bound.

In the experiments so far, the algorithms have estimated 3 parameters $(\lambda, \kappa$ and $b)$ and in the case of the $l_{\infty}$-method also noise. To test how the algorithms behave in a simpler case, i.e. with fewer perturbations, we isolate one or more perturbations.

First, we repeatedly omit one of the perturbations. The results of simulations for the isolation of $\pi_{1}, \pi_{2}$ and $\pi_{3}$ are respectively depicted in Fig. 3 to Fig. 5, which shows tighter bounds for both methods, and better tracking of uncertainties for the MILP-method. We finally isolate two perturbations and estimate the third. Fig. 6 shows tighter bounds for the uncertainties in both methods and the best tracking for the MILP-method.

In purpose to check how the methods behave for perturbations with non-spiky derivative, we consider sinusoid perturbations. The results for the MILP- and $l_{\infty}$-algorithms are depicted in Fig. 7, which shows a good tracking of sinusoid uncertainties and tighter upper bounds for the MILP-method.

Even though the $l_{\infty}$-optimization method sometimes produces better bounds for the perturbations than the MILPoptimization method (Table I), the less sensitivity to the changing of parameters (compare the results in Table II), smaller sensitivity to the increasing of noise (Table III), better tracking and estimation of perturbations in MILP method, and more realistic parameter estimates compared to the rapid change of the $l_{\infty}$-optimization method, makes the MILP method a better candidate for finding acceptable uncertainty bounds. However, the disadvantage of the MILPoptimization method is the longer run time of it in comparison with the $l_{\infty}$-optimization method.

\section{CONCLUSIONS AND FUTURE WORK}

Two optimization methods are presented to estimate bounds of time-varying parameter uncertainty in state-space system. An overhead crane was used as illustrative example.

The promising results of MILP-method shows that exploiting the non-randomness of the perturbations is a viable approach to the estimating time-varying parameter uncertainty. 


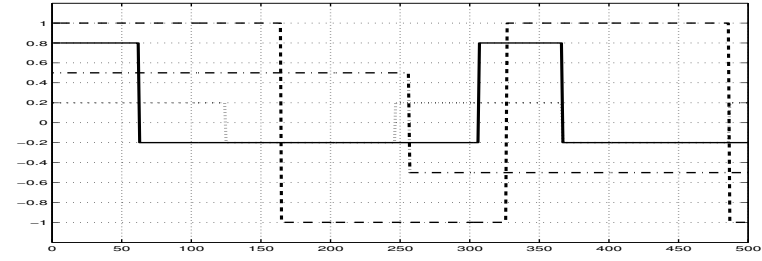

(a)

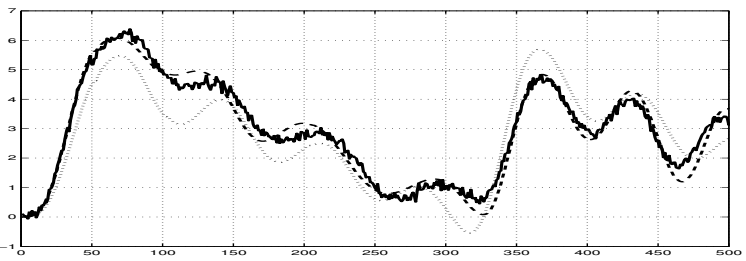

(b)

Fig. 1. (a) Input signal $u_{c}$ (solid line), $\pi_{1}(.),. \pi_{2}(--)$ and $\pi_{3}(-$.$) . (b) Output$ of model(solid line) and output of process (- -) with $\epsilon=0.1$

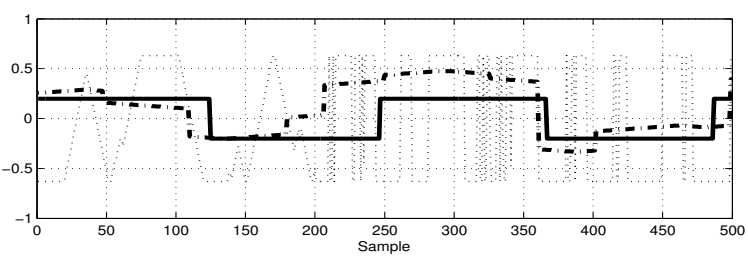

(a)

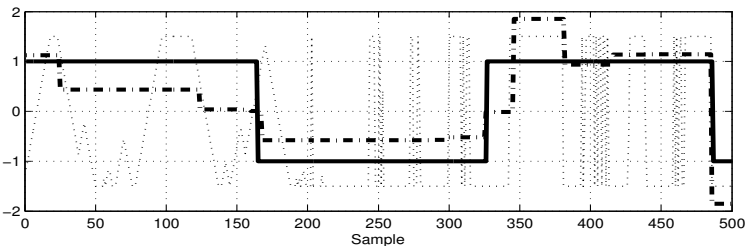

(b)

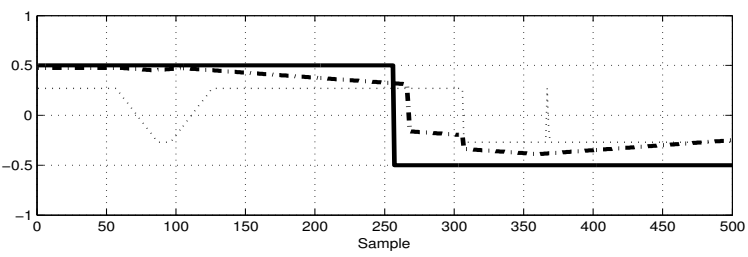

(c)

Fig. 2. Estimates of perturbations $\pi_{1}, \pi_{2}$ and $\pi_{3}$ in (a), (b) and (c), respectively. True perturbations (solid line), $l_{\infty}$-method (...) and MILPmethod (-.-).

TABLE I

ESTIMATED UPPER-BOUNDS OF PERTURBATIONS FOR $\lambda_{0}=3.3$,

$$
\kappa_{0}=0.25 \text { AND } b_{0}=2
$$

\begin{tabular}{|c|c|c|}
\hline $\begin{array}{c}\text { True upper-bound } \\
\text { for perturbation }\end{array}$ & $\begin{array}{c}\text { Upper-estimated } \\
\text { bound for } l_{\infty} \text {-method }\end{array}$ & $\begin{array}{c}\text { Upper-estimated } \\
\text { bound for MILP-method }\end{array}$ \\
\hline$[0.2,1.0,0.5]$ & {$[0.60,1.40,0.27]$} & {$[0.48,1.8,0.5]$} \\
\hline$[0.4,1.0,0.5]$ & {$[0.90,1.75,0.25]$} & {$[0.95,2.0,0.5]$} \\
\hline$[0.2,2.0,0.5]$ & {$[0.70,2.20,0.30]$} & {$[0.70,2.4,0.5]$} \\
\hline$[0.2,1.0,1.0]$ & {$[0.60,1.25,0.70]$} & {$[0.80,1.8,1.0]$} \\
\hline
\end{tabular}

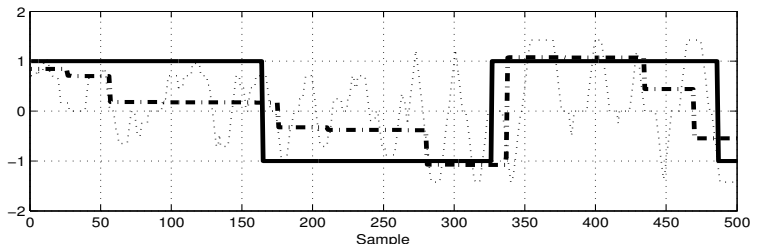

(a)

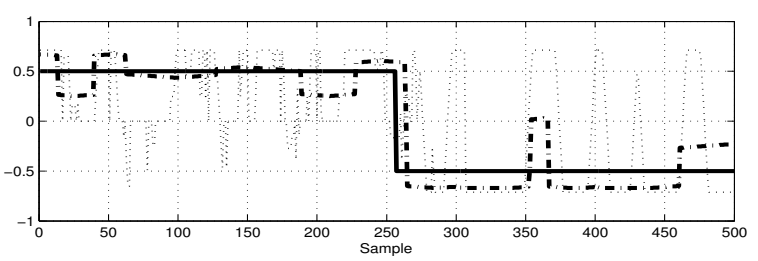

(b)

Fig. 3. Estimates of perturbations $\pi_{2}$ and $\pi_{3}$ in (a), (b), respectively. True perturbations (solid line), $l_{\infty}$-method (...) and MILP-method (-.-).

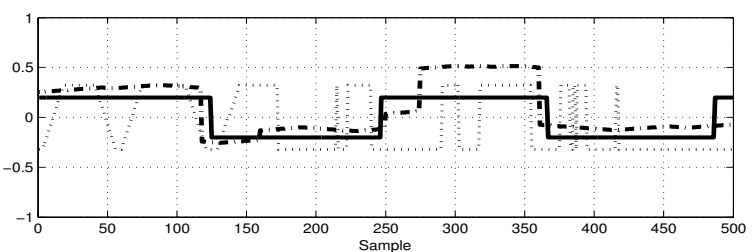

(a)

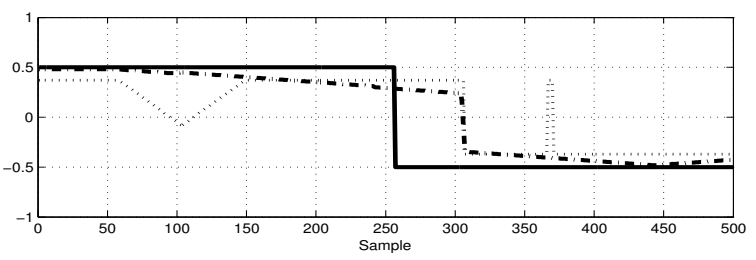

(b)

Fig. 4. Estimates of perturbations $\pi_{1}$ and $\pi_{3}$ in (a), (b), respectively. True perturbations (solid line), $l_{\infty}$-method (...) and MILP-method (-.-).

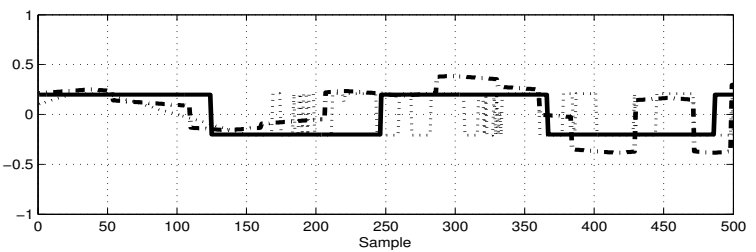

(a)

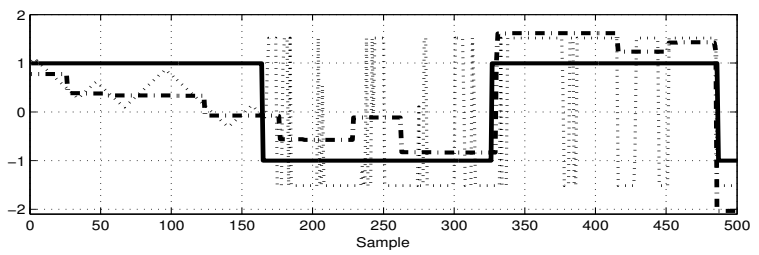

(b)

Fig. 5. Estimates of perturbations $\pi_{1}$ and $\pi_{2}$ in (a), (b), respectively. True perturbations (solid line), $l_{\infty}$-method (...) and MILP-method (-.-). 
TABLE II

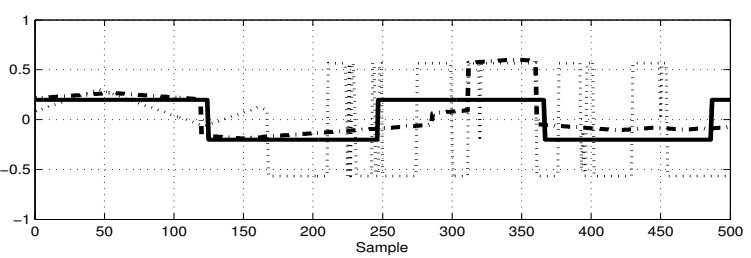

(a)

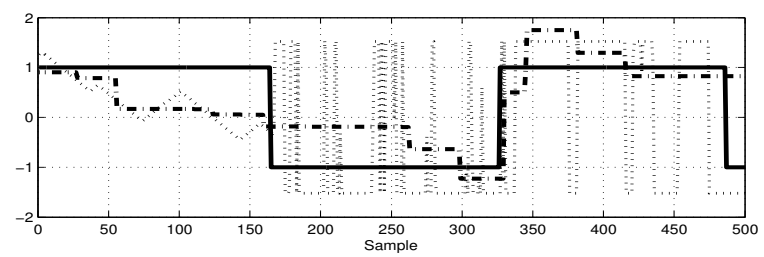

(b)

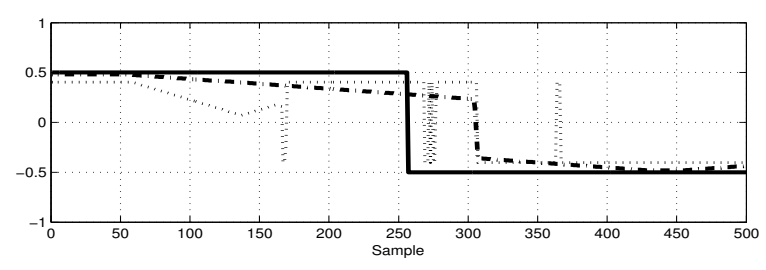

(c)

Fig. 6. Estimates of one perturbation, when the other two perturbations are isolated. $\pi_{1}, \pi_{2}$ and $\pi_{3}$ for the two methods in (a), (b) and (c), respectively. True perturbations (solid line), $l_{\infty}$-method (...) and MILP-method (-.-).

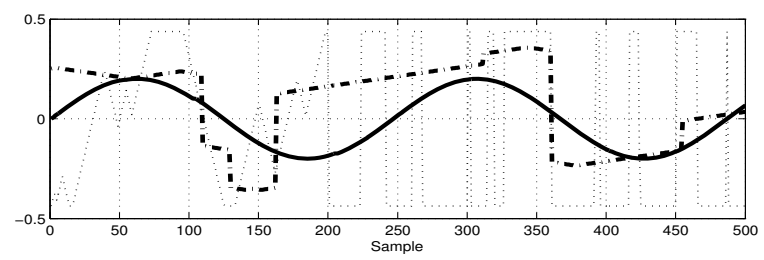

(a)

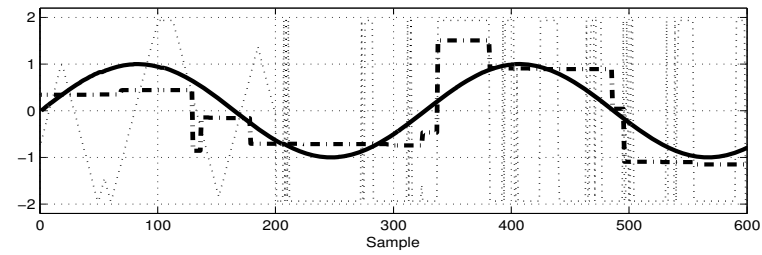

(b)

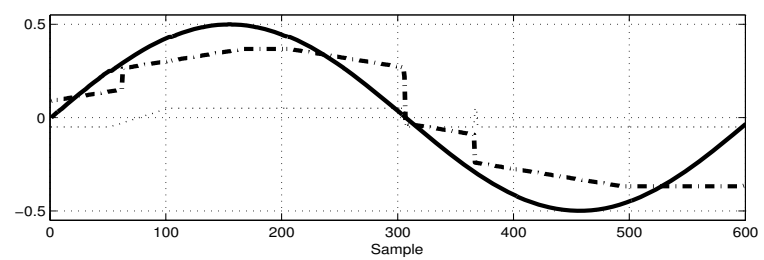

(c)

Fig. 7. The estimation of sinusoid perturbations $\pi_{1}, \pi_{2}$ and $\pi_{3}$ in (a), (b) and (c), respectively. True perturbations (solid line), $l_{\infty}$-method (...) and MILP-method (-.-).
THE SENSITIVITY OF UPPER-ESTIMATED BOUNDS TO DIFFERENT VALUES OF $\lambda_{0}, \kappa_{0}$ AND $b_{0}$, TRUE UPPER-BOUND IS $[0.2,1.0,0.5]$

\begin{tabular}{|c|c|c|}
\hline$\left[\lambda_{0}, \kappa_{0}, b_{0}\right]$ & $\begin{array}{c}\text { Upper-estimated } \\
\text { bound for } l_{\infty} \text {-method }\end{array}$ & $\begin{array}{c}\text { Upper-estimated } \\
\text { bound for MILP-method }\end{array}$ \\
\hline$[3.0,0.25,2.0]$ & {$[0.70,0.70,0.26]$} & {$[0.320 .900 .47]$} \\
\hline$[3.7,0.25,2.0]$ & {$[0.48,1.90,0.35]$} & {$[1.60,1.50,0.50]$} \\
\hline$[3.3,0.20,2.0]$ & {$[0.85,1.50,0.23]$} & {$[0.60,1.50,0.50]$} \\
\hline$[3.3,0.15,2.0]$ & {$[1.70,3.80,0.00]$} & {$[0.50,1.30,0.43]$} \\
\hline$[3.3,0.25,1.5]$ & {$[0.40,1.60,0.30]$} & {$[0.37,1.38,0.47]$} \\
\hline$[3.3,0.25,2.5]$ & {$[0.90,1.60,0.20]$} & {$[0.63,1.70,0.50]$} \\
\hline
\end{tabular}

TABLE III

THE SENSITIVITY OF UPPER-ESTIMATED BOUNDS TO THE CHANGING OF $\epsilon$, TRUE UPPER-BOUND IS $[0.2,1.0,0.5]$

\begin{tabular}{|c|c|c|}
\hline $\begin{array}{c}\text { Window norm } \\
\text { of white noise }\end{array}$ & $\begin{array}{c}\text { Upper-estimated } \\
\text { bound for } l_{\infty} \text {-method }\end{array}$ & $\begin{array}{c}\text { Upper-estimated } \\
\text { bound for MILP-method }\end{array}$ \\
\hline 0.05 & {$[0.4,1.5,0.30]$} & {$[0.40,1.1,0.50]$} \\
\hline 0.10 & {$[0.6,1.4,0.27]$} & {$[0.48,1.8,0.50]$} \\
\hline 0.20 & {$[0.5,1.0,0.30]$} & {$[0.40,1.6,0.45]$} \\
\hline 0.30 & {$[0.5,1.2,0.30]$} & {$[0.40,1.6,0.40]$} \\
\hline
\end{tabular}

For future research, other properties of noise and perturbations may also be exploited, e.g. that the noise is expected to be random and uncorrelated with the perturbations.

\section{ACKNOWLEDGMENTS}

The authors wish to thank Hjalmar Lundbohm Research Center (HLRC) funded by LKAB for financing this research.

\section{REFERENCES}

[1] L. Giarré, M. Milanese and M. Taragna, $H_{\infty}$ identification and model quality estimation. IEEE Transaction on Automatic Control, 42(2): 188199, 1997.

[2] P.M. Mäkilä, J.R. Partington and T.K. Gustafsson, Worst-case controlrelevant identification. Automatica, 31(12): 1799-1819, 1995.

[3] M. Milanese and M. Taragna. $H_{\infty}$ set membership identification: A survey. Automatica, 41: 2019-2032, 2005.

[4] W. Reinelt, A. Garulli and L. Ljung. Comparing different approaches to model error modeling in robust identification. Automatica, 38:787-803, 2002.

[5] V.F. Sokolov. Control oriented model validation and errors quantification in the $l_{1}$ setup. IEEE Transactions on Automatic Control, 50(10): $1501-1508,2005$

[6] A. Johansson, M. Bask and T. Norlander. Dynamic threshold generators for robust fault detection in linear system with parameter uncertainty. Automatica 42: 1095-1106, July 2006.

[7] S. Boyd and L. Vandenberghe. Convex Optimization.Cambridge University, 2007 Stephen Boyd (with Lieven Vandenberghe, 2004)

[8] J. Löfberg. YALMIP : A Toolbox for Modeling and Optimization in MATLAB. In Proceedings of the CACSD Conference, Taipei, Taiwan, 2004.

[9] Carlos E. de Souza, K. A. Barbosa and A. Trofino. Robust filtering for linear systems with convex-bounded uncertain time-varying parameters. IEEE Transactions on Automatic Control, 52(6): 1132-1138, 2007.

[10] D. Xie and G. Xu. Computation of performance robustness bounds for control systems with parameter uncertainties: An LMI approach. IEE Proceeding on Control Theory Application, 152(6): 675-682, 2005.

[11] A. Johansson. Validation of state-space models with time-varying parameter uncertainty. In Proceedings of the $46^{\text {th }}$ IEEE Conference on Decision and Control, New Orleans, USA, 2007.

[12] Louis L. Scharf. Statistical signal processing: Detection, estimation and time series analysis. Addison- Wesley publishing company, 1991.

[13] A. Johansson. Shift-invariant signal norms for fault detection and control. System and Control letters, 57, 105-111, 2008. 